\title{
Voltage Stability Assessment of a Power System Incorporating Wind Turbine Using Power System Analysis Toolbox (Psat)
}

\author{
S. Subbu Lakshmi
}

\begin{abstract}
As power systems become more complex and heavily loaded, voltage stability becomes an increasing serious problem. Voltage instability problems increasing day by day because of demand increase. It is very important to analyze the power system with respect to voltage stability. At present, wind power energy is increasingly penetrating electrical grids. This penetration is mainly driven by better wind technologies. The control capabilities of these new technologies are continuously improving to satisfy grid code requirements, ensuring a safe operation under normal and fault conditions. Double feed induction generators (DFIGs) and Squirrel Cage induction generators (SCIGs) are practically used. When the penetration of wind generation is high, it is important to keep these generators on line as much as possible during grid disturbances. Therefore, there is a significant interest in investigating the dynamic performance and characteristics of the system under high penetration of wind generation. To avoid this voltage collapse and to simplify the study, PSAT will be used to obtain the power flow results. This paper presents a comparative stability analysis of conventional synchronous generators and wind farms based on Squirrel Cage induction generators (SCIG). Based on an appropriate SCIG wind generator model, PV curves, and trained using Artificial Neural Network (ANN) are used to study the effect on system stability of replacing conventional generation by SCIG-based wind generation on the IEEE 14-bus benchmark system. Power System Analysis ToolBox (PSAT), Open Source MATLAB software which is used to obtain the voltage stability for various wind velocities. The voltage stability limits are derived in terms of maximum real power demand.

Various case studies are analyzed.The results obtained using PSAT are given as the training data to the Artificial Neural Network(ANN). The ANN is trained using Back Propagation Algorithm (BPN) algorithm. The trained Network is used to predict the voltage stability limit at a particular wind velocity. PSAT is well suited for this kind of problem as it has the merit of solving both static and dynamic algorithm, exploiting network topology and to extract its component data and it has ability to define user defined models. Artificial Neural Network with BPN technique is preferred here for its advantages including the ability to detect all possible interactions between predictor variables and the availability of multiple training algorithms.

Keywords: Squirrel Cage induction generators (SCIG), Artificial Neural Network (ANN), Wind turbine, Voltage Collapse, Power System Analysis ToolBox (PSAT), Maximum Loadability Limit.
\end{abstract}

\section{INTRODUCTION}

Voltage stability is a fundamental component of dynamic security assessment and it has been emerged as a major concern for power system security and a main limit for loading and power transfer. Voltage stability is usually expressed in term of stability margin, which is defined as the difference between loadability limit and the current operating load level. By means of calculating the critical point, the loading margin to voltage collapse can be determined. PSAT is a MATLAB toolbox for electric power system analysis and control. The continuation power flow method of getting the critical point by tracing the PV curve has been applied to overcome this difficulty. Power System Analysis Toolbox (PSAT) in tracing the PV curve for obtaining Maximum Loading Point (Pmax) involves the input from Wind turbine modeling with IEEE 14-bus benchmark System. It is difficult to realize real-time voltage stability assessment as the calculation speed of this method is slow for power systems with high dimension. Modeling of Wind turbine using the PSAT simulation, a nominal wind speed of $10 \mathrm{~m} / \mathrm{s}$ is used. Air density considered is $1.225 \mathrm{Kg} / \mathrm{m} 3$ under normal conditions. Other parameters such as wind model type (i.e. Weibull, Measurements, and Composite) is needed. By varying the velocities and pitch angle of the wind turbine, Maximum Loadability limit points are obtained. Artificial Neural Network (ANN), a method based on statistics learning theory, is a machine learning algorithm of the new era. . Its main advantages are easy handling, fast speed of approaching and a good graphic presentation of results.

\section{Problem Formulation AND IMPLEMENTATION}

Wind energy is a source of renewable energy which comes from air current flowing across the earth's surface. Wind turbines harvest this kinetic energy and convert it into usable power which can provide electricity for home, farm, school or business applications on small (residential) or large (utility) scales such as industries. 
Wind energy is one of the fastest growing sources of new electricity generation in the world today. These growth trends can be linked to the multi-dimensional benefits associated with wind energy. Thus Modeling wind turbine plays a vital role in extracting the maximum penetration of wind energy. Using Back Propagation [BPN] Algorithm [It requires a dataset of the desired output for many inputs The goal of this algorithm is to find a function that best maps a set of inputs to its correct output].This method is useful in analyzing the stable or unstable points of Voltage variation by mapping the inputs of Pmax (Maximum Loadability Limit) to the correct output of $(0$ or 1$)$ or $(+1$ or -1$)$. Simulink Diagram of Neural Network and the Points of Regression results obtained from the Trained network represents the Stable and unstable Points in the system whether it may be $(+1$ or -1$)$ or $(0$ or 1$)$, thus helps in analyzing the Voltage Stability.

The idea of PSAT and NNTOOL is implemented here for voltage stability. PSAT is used for the benefits of Graphical User Interface other than Mathematical Models as it one-line network diagram editor,GUIs for settings system and routine parameters, User defined model construction and installation, GUI for plotting results ,Filters for converting data to and from other formats, Easily to draw some renewable models.

\section{VOLTAGE STABILITY}

Characteristics of power system to remain in a state of equilibrium. Voltage instability is closely related to the maximum loadability of a transmission network. In present-day power systems, this may take place as a precursor to the traditional frequency instability problem. The main problem here is that the maximum loading of the transmission system is not a fixed quantity. Such quantity depends on the network topology, generation and load patterns and the availability of VAR resources. All these factors can vary with time due to scheduled maintenance, unexpected disturbances, etc.

Voltage problems are expected when developing power systems, and in case of major system breakups. As Voltage stability is a dynamic phenomenon, thus there should be an obvious need for dynamic voltage stability analysis. For the faster transient phenomena, dynamic simulations are necessary, but for slower, longer term phenomena, steady-state based methods might suffice.

\section{The Challenge}

When the penetration of wind generation is high, it is important to keep these generators on line as much as possible during grid disturbances. Therefore, there is a significant interest in investigating the dynamic performance of renewable energy such as Wind due to its Variation in wind speed (Velocity) and characteristics of the system under high penetration of wind generation. The figure below is taken from the case study related to renewable energy resources. Thus the aim here is to achieve the $20 \%$ penetration of wind using PSAT wind turbine modeling.

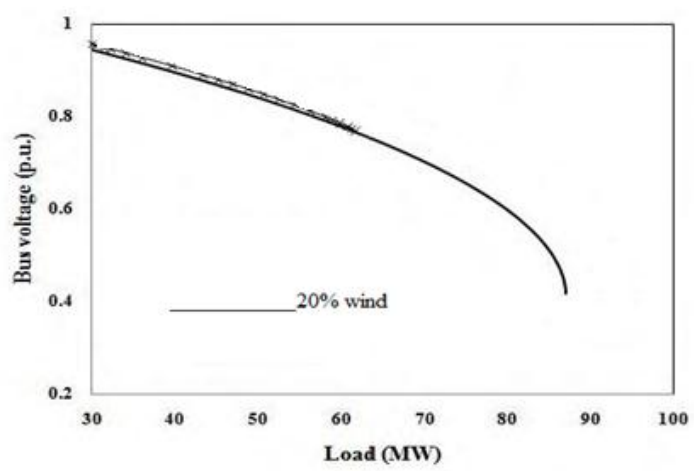

And by Varying Wind Velocities, Maximum Loadability Limit points are obtained. These are helpful in training the neural network tool to analyze various stable points. Here the Voltage Stability analysis is of main concern. This is to analyze the power system to identify certain points of stability by making use of Network topology.

\section{Outline Of Our Paper}

WIND TURBINE MODELLING IN PSAT: Wind turbine is modeled and it has been connected in IEEE 14 bus test system under study.

CPF METHOD: CPF method has been applied to get the critical point by tracing the PV curve.

LOADABILITY LIMIT PREDICTION: Maximum loading point or the voltage stability limit is to be determined to avoid voltage collapse and to maintain the safety operation of electric power systems. It is necessary to evaluate the distance between the current operating state and the voltage critical point in order to escape from the voltage collapse. By means of calculating the critical point, the loading margin to voltage collapse can be determined. 
ANN IMPLEMENTATION: ANN, a mathematical model or computational model that tries to simulate the structure and to model complex relationships between inputs and outputs.

ANN OPERATION : The use of feature reduction techniques can decrease the number of features required and thus reduce the number of system quantities needed to be measured and transmitted.

REGRESSION MODEL: Function approximation model, Simulink model from the trained network shows the output value which corresponds with the input of Maximum Loadability Limit Values. Thus the stable and unstable points are correctly identified.

RESULTS: The empirical results show that fast performance and accurate evaluation of voltage stability indicators have been obtained.

\section{Modelling Of Wind Turbine}

In the wind block of PSAT, window in that helps to easy analyze and modify all parameters related to the wind behaviour. To run this simulation, a nominal wind speed of $17 \mathrm{~m} / \mathrm{s}$ is used. Air density considered is 1.225 $\mathrm{Kg} / \mathrm{m} 3$ under normal conditions.Wind model type "Weibull" is used. Other parameter or data will be given forward. The best thing about simulation on PSAT is the Graphic User Interface that helps easily to draw some renewable models. PSAT graphic model is done for constant speed wind turbine CSWT, with squirrel cage induction generator connected to a infinite bus.

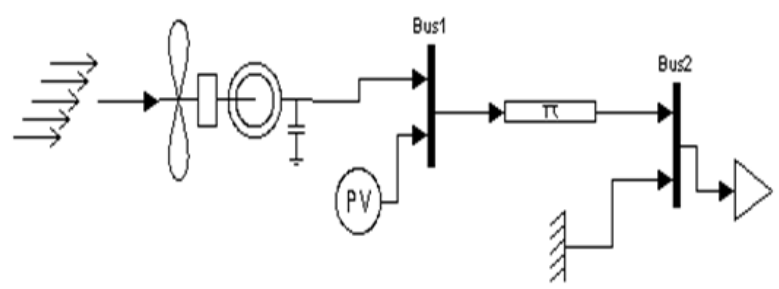

This power system shows from left to right: the natural resource: wind, a wind turbine model connected to bus 1. In the same bus, a PV block model is required for PSAT. This PV model does not work as other generator, it is necessary to imposed the desired voltage and active power at the machine bus. Then a pi representation of the transmission line connects both buses. Finally, the load bus 2 is setup with a load constant power and a slack bus required for PSAT. A PQ block defines a constant power load and Slack generators are modeled as fixed voltage magnitude and phase buses, only one slack bus per network can be defined. At the end of the initialization procedure, the PV and/or slack generators connected at the generator buses are removed. In the Generator PSAT Model, Squirrel cage induction generator, SCIG is widely used for constant or fixed speed wind turbine. The Generator Window Block Mask allows modifying some basic parameters such as power, voltage and frequency ratings (MVA, kV, Hz), stator and rotor resistances and reactances (in p.u.), Inertia Constants, numbers of poles and gear box ratio. Active power can be modified or set on window block. Finally blade length and number of poles are taking from the technical brochure or manufacturer data.

Using the simulink and the PSAT graphic interface, model is open or selected. The Weibull model type is confirmed. The power flow is the first procedure made by the software. It must be run to initialise all variables. First a Power Flow analysis must be done to initialize and data is loaded. PSAT used the Newton-Raphson method for power flow computation. One way of generating electricity from renewable sources is to use wind turbines. A tendency to erect more wind turbines can be observed. As a result of this, in the near future wind turbines may start to influence the behavior of electrical power systems. To investigate the impact of increasing wind turbine penetration, power system dynamics studies need to be carried out. To this end, power system dynamics simulation software is used, in which wind turbine models must be integrated to enable the investigation of increasing wind turbine penetration on power system behavior. If wind turbine models are to be integrated into power system dynamics simulation software, it must be possible to calculate the initial conditions of the dynamic model from load flow data to be able to initialize the dynamic simulation correctly. For the learning procedure in classification, the learning samples (positive and negative) should be prepared, and each sample's class needs to be predefined. In the problem of voltage stability classification, the aim is to identify voltage stability of the time series in each sample (or each window). For this purpose, a reference to Pmax stablility was chosen in each time trajectory. If a set of data in a window is all below the stable chosen reference, the system is stable; otherwise, unstable. The direction of the data towards or away the chosen reference indicates the direction of trajectory of system state condition. The variation of wind velocity in the wind turbine at the relevant buses in the IEEE14 bus test system is chosen for training and the classifier is either stable $(+1)$ or unstable $(-1)$. 


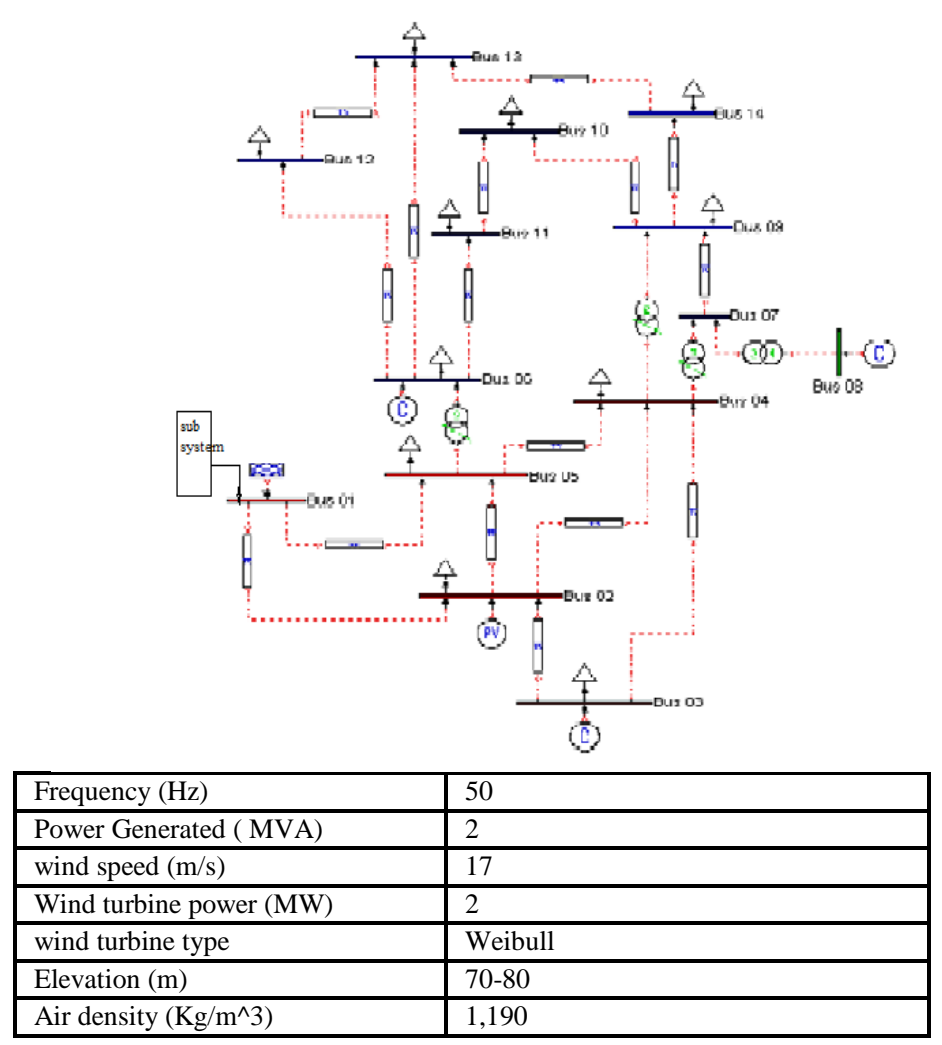

\section{Neural Network Operation For Voltage Stability Analysis}

Maximum loadability limit values (Pmax) are collected and these values are given into the Neural Network for training purpose. Results obtained the neural network such as regression, its performance plot, training states are analysed. The Trained Neural Network Block in the simulink is taken over for Voltage stability analysis.

\section{TRAINED NETWORK SIMULINK MODEL}

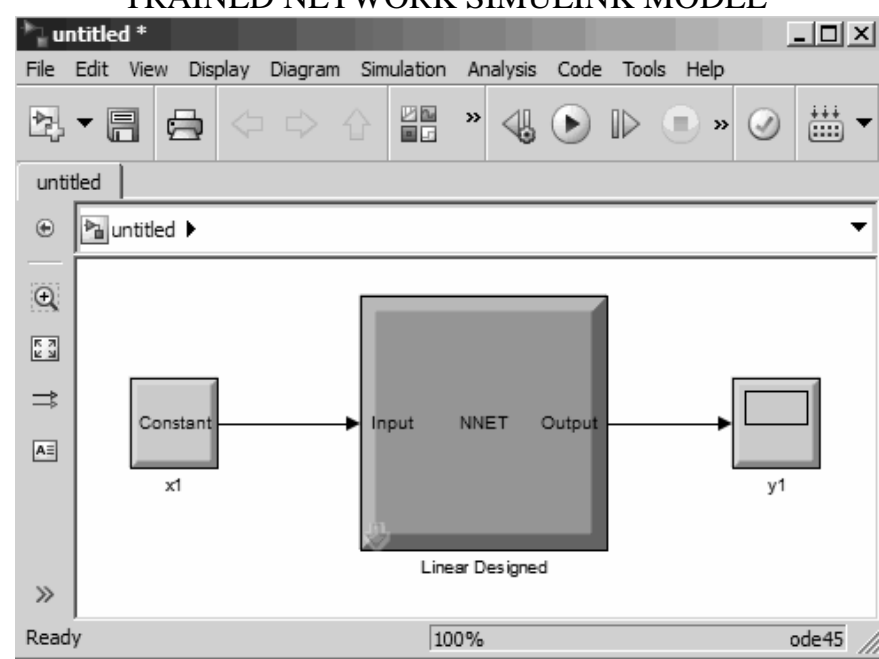

Testing the network can be done with constant block parameters. To test the network, double-click the input Constant $\mathrm{x} 1$ block on the left. 


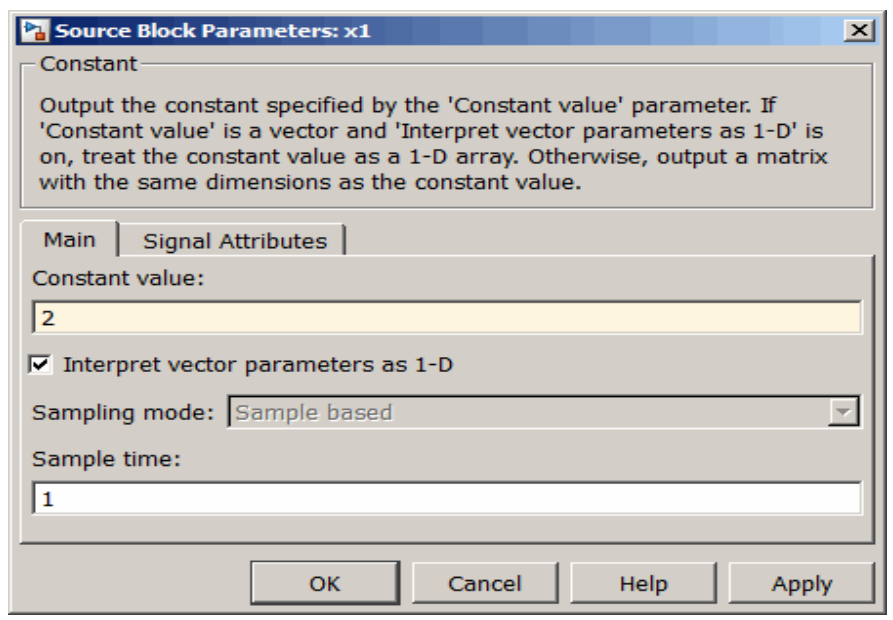

The input block is actually a standard Constant block. Change the constant value from the initial randomly generated value to 2, and then click OK. Select the menu option Simulation > Run. Simulink takes a moment to simulate the system. When the simulation is complete, double-click the output y1 block on the right to see the following display of the network's response.If the output obtained is 3, which is the correct output for an input of Pmax which will be either as Stable or Unstable(0 or 1) or (+1 or -1$)$. By Changing the Input Signal, replace the constant input block with a signal generator from the standard Simulink Sources blockset. Simulate the system and view the network's response. And by Using a Discrete Sample Time Recreate the network, but with a discrete sample time of 0.5 , instead of continuous sampling using the parameter "gensim". For Eg., gensim(net,0.5) and upto many values. Again, by replacing the constant input with a signal generator. Simulating the system and by viewing the network's response stable points and unstable points can be analyzed. The function gensim generates block descriptions of networks. gensim(net,st) The second argument to gensim determines the sample time, which is normally chosen to be some positive real value. If a network has no delays associated with its input weights or layer weights, this value can be set to -1 . A value of -1 causes gensim to generate a network with continuous sampling.

\section{Results}

IEEE 14 BUS SYSTEM CONTINUATION POWER FLOW-WITHOUT WIND TURBINE

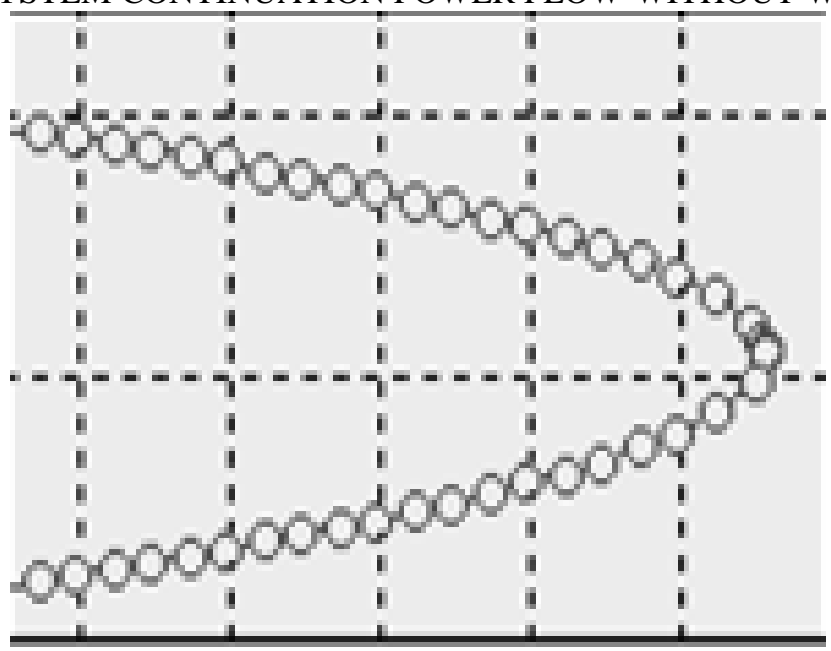


IEEE 14 BUS SYSTEM VOLTAGE PROFILE PMAX VALUE $=11.1214$

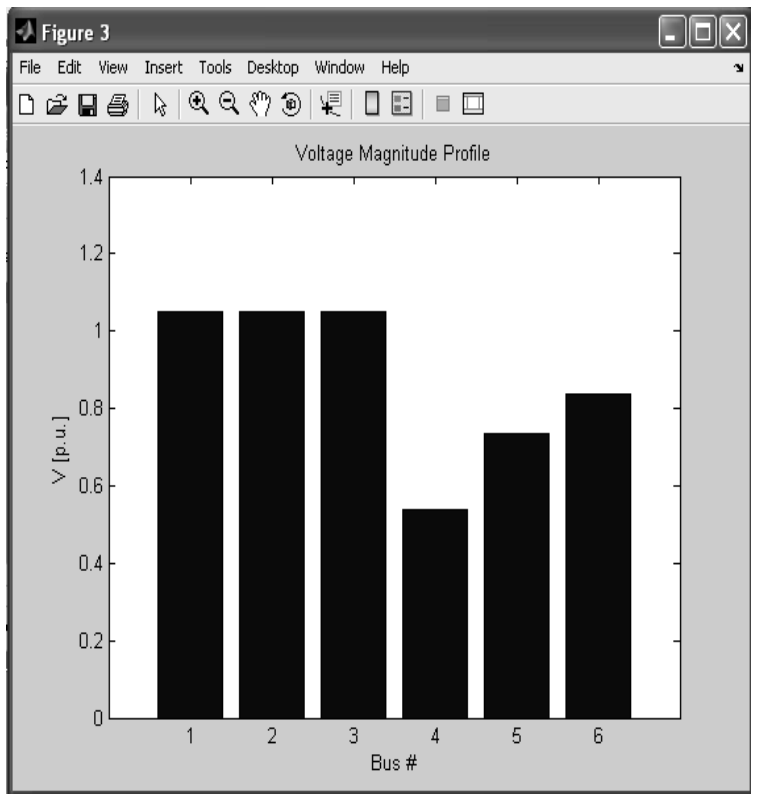

IEEE 14 BUS SYSTEM WITH WIND TURBINE-CONTINUATION POWER FLOW

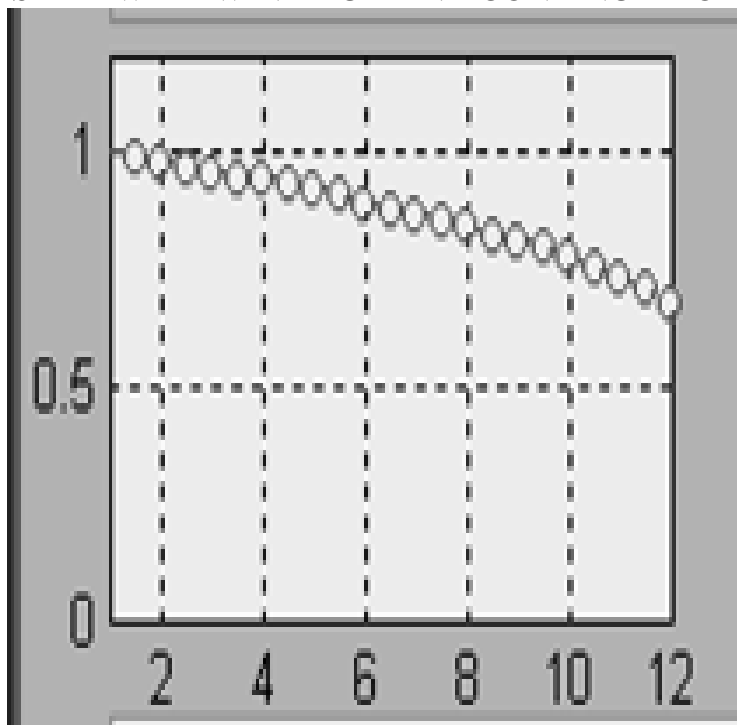

IEEE 14 BUS SYSTEM WITH WIND TURBINE VOLTAGE PROFILE PMAX VALUE $=13.1416$

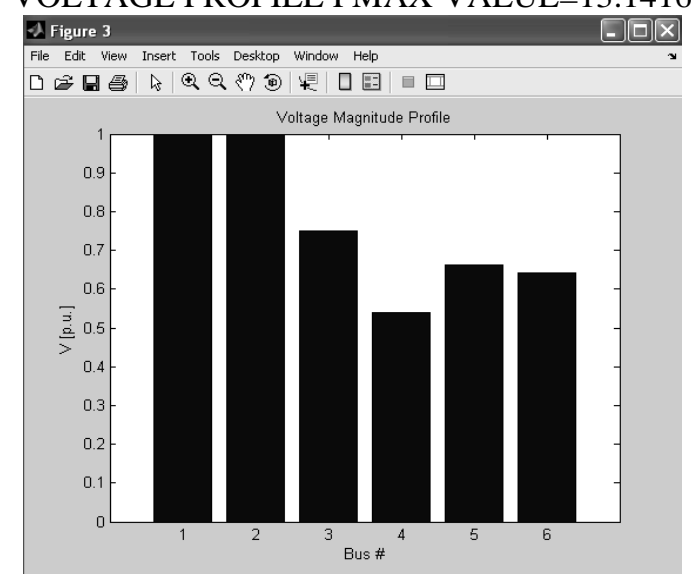

NEURAL NETWORK OUTPUT REPRESENTING STABLE POINT 


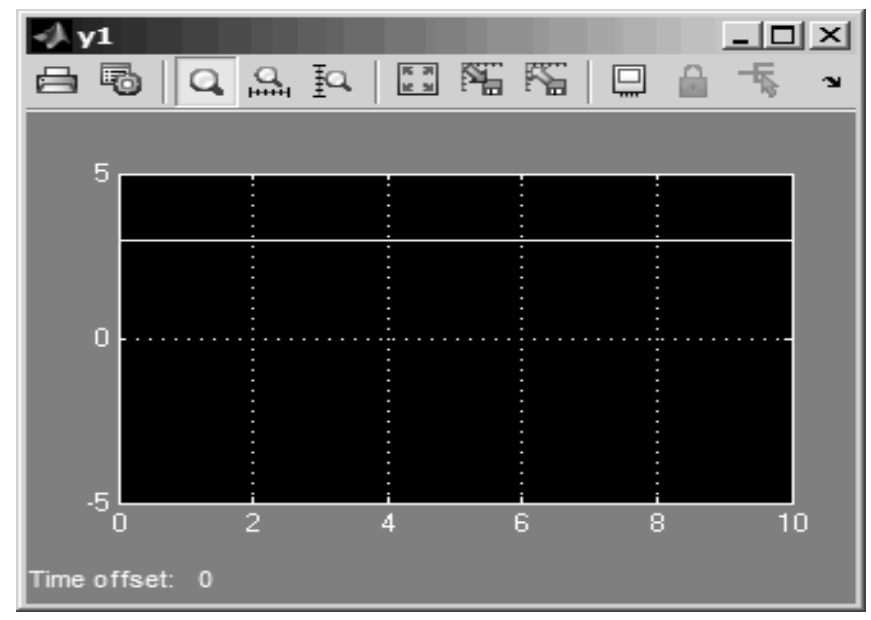

Thus the challenge identified of obtaining the penetration of wind is achieved by modeling using PSAT.And the Maximum Loadability limit values are collected for further Neural Network operation. Maximum loadability limit values (Pmax) are collected and these values are given into the Neural Network for training purpose. Results obtained the neural network such as regression,its performance plot, training states are analysed. The Trained Neural Network Block in the simulink is taken over for Voltage stability analysis.

If the output obtained is 3, which is the correct output for an input of Pmax which will be either as Stable or Unstable( $(0$ or 1$)$ or $(+1$ or -1$)$. By Changing the Input Signal, replace the constant input block with a signal generator from the standard Simulink Sources blockset. Simulate the system and view the network's response.

\section{Conclusion And Future Work}

Thus using Efficient Tool such Power System Analysis Toolbox (PSAT), the penetration of wind energy of about $20 \%$ is achieved and the Neural Network is trained. The trained network identified the voltage stability points of the system under various wind velocities and gives the information about the maximum loading point of the system. Dynamic Performance of any renewable energy methodology shall be investigated in future without any complication using this efficient technique.

\section{References}

[1]. Ali M. Eltamaly, "Modeling of wind turbine driving permanent magnet generator with maximum power point tracking system".

[2]. Sina Lotfi and Mahyar Sajedi "Modeling and application of permanent magnet synchronous generator (PMSG) based variable speed wind generation system", International Journal of the Physical Sciences Vol. 7(3), pp. 370 - 376, 16th January, 2012.

[3]. Federico Milano, "Assessing Adequate Voltage Stability Analysis Tools for Networks with High Wind Power Penetration", IEEE 2008 DRPT Conference, Nanjing, China, pp. 2492-2497,April,2008.

[4]. Ch. Eping, J. Stenzel, M. Paoller, H. Mauller, "Impact of Large Scale Wind Power on Power System Stability".

[5]. Weiwei Miao, Hongjie Jia, "Wind Power System Voltage Stability Assessment and Local Control with Considering Demand Response". 